\title{
CHE COSA NON È DECIDIBILE Cinque regioni del coto vedado
}

WHAT IS NOT DECIDABLE

Five regions of the coto vedado

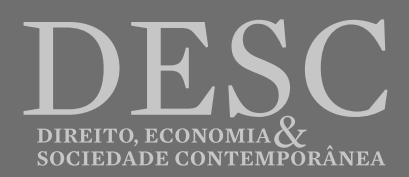




\title{
CHE COSA NON È DECIDIBILE Cinque regioni del coto vedado
}

\author{
WHAT IS NOT DECIDABLE \\ Five regions of the coto vedado
}

MICHELANGELO BOVERO

Università degli Studi di Torino

Conselho Editorial DESC

orcid.org/0000-0002-4590-0526

michelangelo.bovero@unito.it

\begin{abstract}
Riassunto: in questo articolo, l'A. richiama l'attenzione sul concetto di «sfera dell'indecidibile» coniato da Luigi Ferrajoli, ponendolo a confronto con la nozione di «coto vedado» di Ernesto Garzón Valdés e con l'analoga idea di «territorio» o «frontiera» inviolabile elaborata da Norberto Bobbio: le tre nozioni indicano l'insieme di principi e regole costituzionali che nessun potere politico può violare negli stati democratici di diritto, al centro del quale si trovano i diritti individuali fondamentali. L'A. propone un'interpretazione estensiva della teoria della democrazia di Bobbio capace di offrire una soluzione più avanzata al problema dei limiti del potere politico democratico. Invita a riconoscere nelle «regole del gioco» indicate da Bobbio le condizioni (in senso logico) della democrazia, articolate in due serie: cinque condizioni formali, contenute nelle regole di competenza e di procedura che riguardano il «chi» e il «come» delle decisioni collettive; e cinque condizioni sostanziali, contenute nei principi normativi impliciti nella «sesta regola» dell'elenco di Bobbio, che prescrivono limiti e vincoli al «che cosa», ossia alla sostanza delle medesime decisioni. Tali condizioni sostanziali corrispondono a quelle che l'A. chiama le «cinque regioni del coto vedado». L'A. torna in conclusione sulla concezione di Ferrajoli, in cui riconosce un miglioramento teorico rispetto alle elaborazioni esplicite sia di Bobbio sia di Garzón Valdés; ma sostiene che la teoria delle condizioni e precondizioni della democrazia ricavata per interpretazione estensiva dalla costruzione teorica di Bobbio offre un miglior fondamento razionale alla costruzione della «sfera dell'indecidibile».
\end{abstract}

Parole-chiavi: democrazia, sfera dell'indecidibile, coto vedado

Abstract: in this article, the author highlights the concept of the "sphere of the
undecidable" coined by Luigi Ferrajoli, comparing it to the notion of "coto vedado"
by Ernesto Garzón Valdés and with the similar idea of inviolable "territory" or
"frontier" elaborated by Norberto Bobbio: the three notions indicate the set of 
principles and constitutional rules that no political power can violate in democratic states, at the center of which individual fundamental rights are found. The author proposes an extensive interpretation of Bobbio's theory of democracy capable of offering a more advanced solution to the problem of the limits of democratic political power. The author invites to recognize in the "game rules" indicated by Bobbio the conditions (in a logical sense) of democracy, divided into two series: five formal conditions, contained in competence and procedure rules concerning "who" and "how" of collective decisions; and five substantive conditions, contained in the normative principles implicit in the "sixth rule" of Bobbio's list, which prescribe limits and constraints to "what", the substance of the same decisions. These substantial conditions correspond to those that the author calls the "five regions of the coto vedado". In conclusion, the author returns on the concept of Ferrajoli, in which he recognizes a theoretical improvement over the explicit elaborations of both Bobbio and Garzón Valdés; but he maintains that the theory of the conditions and preconditions of democracy obtained through extensive interpretation from Bobbio's theoretical construction offers a better rational foundation for the construction of the "sphere of the undecidable".

Keywords: democracy, sphere of the undecidable, coto vedado

1. Da molto tempo, tra gli studiosi di teoria giuridica e politica, e non solo tra quelli di madre-lingua spagnola, è divenuto usuale ricorrere all'espressione castigliana coto vedado. Com'è noto, si tratta di una grande invenzione - o meglio, re-invenzione - linguistica di Ernesto Garzón Valdés. ${ }^{1}$ Con tale formula si indica un insieme di principi e/o regole basilari, di rango costituzionale, che nessun potere politico costituito negli stati democratici di diritto, neppure quello della maggioranza, può violare. Al centro del coto vedado si trovano i diritti individuali fondamentali.

Nell'opera di Norberto Bobbio compare un'espressione simile, con un significato analogo: «territorio» o «frontiera» inviolabile, che costituisce un limite invalicabile all'applicazione del principio di maggioranza. ${ }^{2}$ Conviene citare per intero un passo della Teoria generale della politica di Bobbio:

1 Così la presenta lo stesso Garzón Valdés in un denso scritto autobiografico, intitolato Para ir terminando e compreso in M. Atienza, El derecho como argumentación, Fontamara, México D.F., 2003: «Hace ya muchos años que vengo insistiendo en la necesidad conceptual de incluir en todo diseño democrático-representativo lo que suelo llamar "coto vedado", expresión tomada del título de una obra de Juan Goytisolo, compañero de mis años madrileños a comienzo de los cinquenta del siglo pasado. Al recurrir a esta expresión me pareció que no sólo introducía una referencia literaria en el vocabulario jurídico-político sino que, además, me servía adecuadamente para denotar un campo en el que debía estar prohibido el ingreso de la política» (p. 43).

2 Che il significato attribuito da Bobbio a queste espressioni sia analogo a quello di coto vedado lo riconosce lo stesso Garzón Valdés nel saggio El futuro de la democracia. Problemas conceptuales y empíricos: algunas propuestas de Norberto Bobbio, in L. Córdova Vianello, P. Salazar Ugarte (coordinadores), Política y derecho. (Re)pensar a Bobbio, Unam \& Siglo ventiuno editores, México D.F. 2005: «Bobbio recurre también a una metáfora geográfica (similar a la del coto) cuando se refiere a la inviolabilidad de los derechos del hombre y del ciudadano establecida en todas las constituciones liberales» (p. 112-13). 
Tutte le costituzioni liberali sono caratterizzate dall'affermazione di diritti dell'uomo e del cittadino che vengono detti "inviolabili": ora, l'inviolabilità consiste proprio in questo, che essi non possono essere limitati e tanto meno soppressi da una decisione collettiva anche presa a maggioranza. Proprio per questa loro inattaccabilità da parte di una qualsiasi decisione maggioritaria, tali diritti sono stati chiamati diritti contro la maggioranza, e vengono in talune costituzioni anche garantiti giuridicamente mediante il controllo costituzionale delle leggi (cioè delle decisioni prese a maggioranza) e la dichiarazione della illegittimità delle leggi che non li rispettano. La vasta sfera dei diritti di libertà può essere interpretata come una specie di territorio, di frontiera, di fronte a cui si arresta la potenza del principio di maggioranza. ${ }^{3}$

Più recentemente, Luigi Ferrajoli ha coniato una formula nuova per indicare il medesimo concetto, o meglio, come cercherò di spiegare nelle conclusioni del presente lavoro, per inquadrare il medesimo problema, predisponendone una soluzione più avanzata: «sfera dell'indecidibile», o «del non decidibile» ${ }^{4}$. Intendo mostrare in che senso la costruzione concettuale di Ferrajoli rappresenta un progresso notevole - anche a questo riguardo - per la teoria del diritto e della democrazia. Al tempo stesso, intendo sostenere che nella teoria della democrazia di Bobbio, o almeno in una interpretazione estensiva (ma credo non forzata) di essa, si trovano elementi per una soluzione del problema sostanzialmente convergente con quella proposta da Ferrajoli.

2. Affronterò la questione dal punto di vista della teoria bobbiana delle regole del gioco democratico. Faccio riferimento all'elenco di sei regole del gioco che si può leggere nel capitolo della Teoria generale della politica intitolato «Dall'ideologia democratica agli universali procedurali». ${ }^{5}$ La tavola bobbiana delle sei regole non è che la sintetica traduzione in norme, o in principi ispiratori di norme, della concezione procedurale della democrazia. Anzi, le sei regole non sono se non l'esplicitazione articolata della sua famosa definizione

3 N. Bobbio, Teoria generale della politica, a cura di M. Bovero, Einaudi, Torino 1999 (d'ora in poi richiamata con la sigla $T G P$ ), pp. 399-400. Il capitolo della $T G P$ da cui traggo la citazione corrisponde al saggio La regola di maggioranza: limiti e aporie, pubblicato originariamente da Bobbio nel 1981 sulla rivista «Fenomenologia e società», IV, n. 13-14.

4 La formula è stata introdotta da Luigi Ferrajoli nel saggio Diritti fondamentali, uscito su «Teoria politica», XIV, n. 2, 1998, pp. 3-33 (v. spec. p. 15), poi ripubblicatro in apertura di L. Ferrajoli (et alii), Diritti fondamentali, a cura di E. Vitale, Laterza, Roma-Bari 2001 (cfr. p. 19). Il concetto è stato poi ripreso ed ampiamente sviluppato in L. Ferrajoli, Principia iuris. Teoria del diritto e della democrazia, 3 voll., Laterza, Roma-Bari 2007: v. in part. vol. I, Teoria del diritto, $\S \S 11.18,12.1$ e 12.6; vol. II, Teoria della democrazia, $\S 13.4,13.8,15.1$ e 16.14 .

$5 T G P$, pp. 370-83; l'elenco delle regole si trova alla p. 381. Questo capitolo della TGP riproduce il testo (sino ad allora inedito) di una conferenza tenuta da Bobbio a Bogotà nel 1987, originariamente intitolata Democrazia ed Europa. Nell'opera di Bobbio si incontrano altre versioni della tavola delle regole del gioco democratico. La differenza tra esse riguarda essenzialmente il numero delle regole elencate nelle diverse occasioni, che varia da due a nove. Ho scelto la versione della conferenza di Bogotà, ora in $T G P$, perché è la più recente e dunque può considerarsi, anche nella specificità delle formulazioni linguistiche, quella definitiva. Faccio comunque osservare che essa riprende, con qualche variante lessicale, l'elenco di regole presentato da Bobbio nel saggio del 1975 Quali alternative alla democrazia rappresentativa?, poi ricompreso in N. Bobbio, Quale socialismo?, Einaudi, Torino 1976 (v. l'elenco alle pp. 42-43); e che le sei regole formulate nel testo di Bogotà, ora in TGP, corrispondono sostanzialmente, ancora una volta con poche varianti, a quelle enumerate dalla terza all'ottava nella voce «Democrazia» redatta da Bobbio per il Dizionario di politica, diretto da N. Bobbio e N. Matteucci, Utet, Torino 1976. 
minima, «secondo cui - come si legge nella Premessa a Il futuro della democrazia - per regime democratico s'intende primariamente un insieme di regole di procedura per la formazione delle decisioni collettive, in cui è prevista e facilitata la partecipazione più ampia possibile degli interessati». ${ }^{6}$ In una serie di lavori più o meno recenti, ${ }^{7}$ ho invitato a riconoscere nelle regole del gioco individuate da Bobbio le condizioni, disgiuntamente necessarie e congiuntamente sufficienti, della democrazia, ridefinita classicamente come il regime dell'eguaglianza e della libertà politiche. Abbiamo imparato dagli antichi, ma poi anche da Kelsen e dallo stesso Bobbio, a chiamare democrazia un regime in cui le decisioni collettive, le norme vincolanti per tutti, non emanano dall'alto, da un soggetto (il monarca o il tiranno) o da pochi soggetti (gli aristocratici o gli oligarchi) che si ergono al di sopra della collettività, ma sono frutto di un processo decisionale che scaturisce dal basso, al quale tutti (i destinatari delle decisioni collettive) hanno diritto di partecipare in modo eguale ed egualmente libero. In questo senso, la democrazia è il regime dell'eguaglianza politica e della libertà politica. Ora: le regole del gioco democratico sono contenute implicitamente nei principi di eguaglianza e libertà politica; ovvero, che è lo stesso, sono riconoscibili come democratiche quelle regole che costituiscono una conseguente esplicitazione dei principi di egualglianza e libertà politica, e per questo tali regole valgono come le condizioni alle quali un regime è (riconoscibile come) democratico, ossia è un regime di eguaglianza e libertà politica. Il gioco politico è democratico se e finché simili regole sono rispettate; se vengono alterate o applicate in maniera scorretta, non coerente con i principi democratici, si comincia a giocare ad un altro gioco. A volte, senza rendersene conto; a volte, intenzionalmente.

3. La prima regola, nell'elenco di Bobbio, pone una condizione di eguaglianza come inclusività: tutti i cittadini passivi, sottoposti all'obbligo politico di obbedire alle norme della collettività, devono essere cittadini attivi, titolari del diritto-potere di partecipare, anzitutto con il voto elettorale, al processo di formazione delle decisioni collettive, senza discriminazioni. La seconda regola pone una condizione di eguaglianza come equivalenza: i voti di tutti i cittadini devono avere peso eguale, nessuno deve contare più o meno di un altro. La terza regola pone una condizione di libertà soggettiva: l'opinione politica di ciascuno deve potersi formare liberamente, in assenza di interferenze distorsive, il che esige almeno che sia garantito

6 N. Bobbio, Il futuro della democrazia, Einaudi, Torino 1984, p. X.

7 M. Bovero, Il concetto di democrazia. Per una ridefinizione radicale, in «Il Ponte», LIX, n. 2, 2003, pp. 68-86; Id., Ma la democrazia ha un futuro? Uno sguardo dall'Italia, in «Ragion pratica», n. 25, 2005, pp. 419-36; Id., Isto é democracia?, in F. Schüler, G. Axt, J. Machado da Silva (eds.), Fronteiras do pensamento. Retratos de um mundo complexo, Editora Unisinos, São Leopoldo (Brasil) 2008, pp. 309-336; Id., La democrazia e le sue condizioni, Notizie editrice, Modena 2009; Id., La democracia y sus condiciones, in «Revista de la Facultad de Derecho de México», LX, 253, enero-junio 2010, pp. 11-30; Id., Democrazia al crepuscolo?, in M. Bovero, V. Pazé (a cura di), La democrazia in nove lezioni, Laterza, Roma-Bari 2010, pp. 3-20. 
il pluralismo dei (e nei) mezzi di informazione e persuasione. La quarta regola pone una condizione di libertà oggettiva: i cittadini devono poter scegliere tra proposte e programmi politici effettivamente diversi tra loro, entro una gamma di alternative sufficientemente ampia da consentire a ciascuno di riconoscersi in un orientamento preciso, il che esige almeno che sia consentito e protetto il pluralismo dei partiti, associazioni e movimenti politici. La quinta regola pone una condizione di efficienza per l'intero processo di decisione collettiva, dal momento elettorale alle deliberazioni degli organi rappresentativi: le decisioni devono essere assunte in base al principio di maggioranza, che è (per Bobbio, semplicemente) una regola tecnica, idonea a superare l'eterogeneità o il contrasto o il conflitto delle opinioni particolari. ${ }^{8}$ Ma è anche, come sappiamo, una regola che ha limiti di applicazione. ${ }^{9}$ Infatti, la sesta e ultima regola dell'elenco che stiamo esaminando stabilisce che: «nessuna decisione presa a maggioranza deve limitare i diritti della minoranza, particolarmente il diritto di diventare a sua volta maggioranza a parità di condizioni». ${ }^{10}$

Questa regola conclusiva - una vera e propria regola di chiusura - non assomiglia alle altre: non è una regola di procedura, non riguarda la forma bensì il contenuto delle decisioni. Ma Bobbio, il «proceduralista» Bobbio, ne è perfettamente consapevole. Basta leggere il commento che segue l'elenco delle regole compreso nella voce «Democrazia» del Dizionario di politica: ${ }^{11}$ «tutte queste regole stabiliscono come si debba arrivare alla decisione politica, non che cosa si debba decidere. Dal punto di vista del che cosa, l'insieme delle regole del gioco democratico non stabiliscono nulla, salvo l'esclusione delle decisioni che in qualche modo contribuirebbero a rendere vane una o più regole del gioco». ${ }^{12}$ Possiamo dunque considerare l'ultima regola dell'elenco di Bobbio come una condizione di salvaguardia o di sopravvivenza della democrazia: il rispetto di questa regola è indispensabile perché si possa continuare a giocare allo stesso gioco. Ma ho fatto notare altre volte che la sua formulazione esplicita appare riduttiva rispetto al significato e alla portata che Bobbio stesso attribuisce a tale regola nel commento citato. Perciò ne suggerisco un'interpretazione estensiva, invitando a considerare implicito nel rispetto dei «diritti della minoranza», che viene imposto dalla sesta

\footnotetext{
8 Com'è noto, Bobbio prende le distanze dalla tesi di Kelsen, che giustifica la regola di maggioranza con l'argomento assiologico secondo cui essa consentirebbe la migliore approssimazione possibile ad un regime di perfetta autonomia, nel quale le decisioni collettive dovrebbero risultare in accordo con la volontà di tutti gli individui consociati. Per Bobbio, la regola di maggioranza è da considerarsi un puro espediente tecnico, migliore di (cioè preferibile a) la regola dell'unanimità semplicemente perché più utile allo scopo di determinare qual è la volontà collettiva. $\mathrm{O}$ almeno, nel giustificare la regola di maggioranza gli argomenti tecnici sono per Bobbio «più probanti» di quelli assiologici. Cfr. TGP, p. 391.

9 Ad essi Bobbio dedica il § 7 del saggio La regola di maggioranza: limiti e aporie, ora in TGP (cfr. sopra, n. 3); inoltre, nel medesimo saggio il $\S 6$ è dedicato ai «Limiti di validità», il $\S 8$ ai «Limiti di efficacia».

$10 T G P$, p. 381.

11 Ricordo che tale elenco è per due terzi sovrapponibile a quello che si trova in $T G P$ : cfr. sopra, n. 5.

12 Nella più recente edizione (N. Bobbio, N. Matteucci, G. Pasquino, Il dizionario di politica, Utet Libreria, Torino 2004) l'elenco e il commento si trovano a p. 241; l'ultimo corsivo è aggiunto da me.
} 
regola a tutte le decisioni della maggioranza, il rispetto di tutte le altre regole e la proibizione di tutte le decisioni che contribuirebbero a renderle vane, cioè ad alterarne il significato di condizioni della democrazia. Così reinterpretata, la sesta regola del gioco pone per la democrazia una condizione di salvaguardia non semplice, bensì articolabile in cinque punti: che corrispondono a quelle che invito a riconoscere come le «cinque regioni del coto vedado». Naturalmente, sono consapevole di non poter attribuire a Bobbio la mia interpretazione di ciò che significa «rendere vane» le regole del gioco. Dunque mi assumo la responsabilità di questo frammento «non puramente procedurale» della teoria della democrazia (credo che questa formula possa piacere a Luigi Ferrajoli), così come della riformulazione di tale teoria in termini di condizioni e precondizioni della democrazia. ${ }^{13}$

4. Traccio qui di seguito un profilo schematico delle «cinque regioni».

a) La sesta regola del gioco vieta anzitutto qualsiasi decisione che possa alterare le altre cinque regole del gioco. Queste sono indisponibili al potere della maggioranza. Ma non solo a quello delle maggioranze politiche contingenti: se si vuol continuare il gioco democratico, le regole che stabiliscono le condizioni della democrazia - cioè le regole costitutive del gioco, che sono tali in quanto riguardano la formazione democratica della volontà politica - devono considerarsi sottratte anche alle alterazioni eventualmente prodotte da una decisione all'unanimità (che si configurerebbe come una sorta di suicidio di massa della democrazia), ${ }^{14}$ e perciò a fortiori indisponibili al potere delle maggioranze qualificate variamente richieste nei diversi ordinamenti per la revisione delle norme costituzionali.

b) Non basta. La sesta regola vieta (implicitamente) qualunque decisione che contribuisca «a rendere vane» una o più regole del gioco. E ciò allarga l'estensione del «terreno proibito» per qualunque potere di maggioranza - ovvero l'ampiezza del coto vedado, o della «sfera dell'indecidibile» - a quei diritti fondamentali che della democrazia costituiscono le precondizioni. Tutti i diritti stabiliti in costituzioni rigide sono per definizione sottratti alla disponibilità delle maggioranze politiche contingenti; ma dallo specifico punto di vista della salvaguardia della democrazia, debbono considerarsi assolutamente indisponibili, e non solo alle maggioranze politiche ma anche al potere di revisione costituzionale, i quattro grandi diritti di libertà dei moderni. ${ }^{15}$ Ossia: la libertà personale, la libertà di opinione e di manifestazione del pensiero, la libertà di riunione, la libertà di associazione. «Questi

\footnotetext{
13 Questi due termini, «condizioni» e «precondizioni», si incontrano raramente e in modo del tutto occasionale nei saggi di Bobbio dedicati alla democrazia. Cfr. ad esempio Il futuro della democrazia cit., pp. 6 e 63.

14 Ernesto Garzón Valdés ammonisce che «librada a sí misma, la democracia tiene una tendencia suicida» (Para ir terminando cit., p. 45). Cfr. anche Id., Instituciones suicidas, Paidós, México D.F., 2000.

15 Così li chiama Bobbio: cfr. ad esempio TGP, p. 304.
} 
diritti», dice Bobbio, «sono il presupposto necessario per il corretto funzionamento degli stessi meccanismi prevalentemente procedurali che caratterizzano un regime democratico. Le norme costituzionali che attribuiscono questi diritti non sono propriamente regole del gioco: sono regole preliminari che permettono lo svolgimento del gioco». ${ }^{16}$ In questo senso si può dire che, se le regole del gioco rappresentano le condizioni della democrazia, i diritti di libertà ne sono le precondizioni indispensabili: in quanto tali, inviolabili e inalterabili, anche da parte del potere di revisione costituzionale, se si vuole evitare che mediante l'esercizio di tale potere vengano poste le premesse per instaurare un regime non più (riconoscibile come) democratico, o una forma di governo che della democrazia conservi solo le apparenze esteriori. Ribadisco: inviolabili in quanto tali, cioè in quanto precondizioni della democrazia; ovvero, non già perché i diritti di libertà siano fini in sé, o principi assoluti, o valori morali, come forse direbbe Ernesto Garzón Valdés; oppure «ragioni sociali» della (di una) società politica stipulate nella sua costituzione, come direbbe Luigi Ferrajoli. Indipendentemente dal fatto che li si voglia qualificare in uno o più d'uno di questi modi (valori assoluti, fini ultimi, ecc.), i diritti di libertà debbono considerarsi inviolabili e inalterabili da parte di qualunque organo decisionale di un ordinamento democratico, perché una loro violazione vanificherebbe la democrazia: senza la garanzia dell'immunità personale per elettori e candidati, senza la garanzia delle libertà di opinione, di riunione, di associazione, una competizione elettorale a suffragio universale non è altro che una messinscena ingannevole, e «la stessa regola della maggioranza diventa un espediente ipocrita». ${ }^{17}$

c) Ma nelle precondizioni della democrazia si devono comprendere anche alcuni determinati diritti sociali: in primo luogo, il diritto all'istruzione, inteso come diritto all'educazione del cittadino, senza la garanzia del quale appare «vano» (vuoto, inutile, privo di senso) assicurare agli individui le condizioni oggettive di una scelta politica libera; in secondo luogo, il diritto alla sussistenza, senza la garanzia del quale si va incontro al pericolo, già additato da Rousseau, che qualcuno ceda alla tentazione di vendere il proprio voto, e sappiamo che troverà sempre qualcun altro disposto a comprarlo. Questi diritti rappresentano, a mio giudizio, le indispensabili precondizioni sociali delle precondizioni liberali della democrazia. Così come i diritti politici di partecipazione democratica risulterebbero vanificati dall'assenza di garanzie per i diritti all'immunità personale, alla libera manifestazione del pensiero, alla libera riunione e associazione; allo stesso modo, le grandi libertà dei moderni rimarrebbero virtuali e svuotate del loro senso politico, ossia private della loro funzione democratica, in

16 Il futuro della democrazia cit., p. 6, corsivi aggiunti.

17 Così afferma Bobbio nel saggio La regola della maggioranza e i suoi limiti, compreso nel vol. collettaneo Soggetti e potere, Bibliopolis, Napoli 1981, p. 18. 
mancanza di garanzie per il diritto all'istruzione, oltre che all'informazione libera e plurale, e ad un minimo assicurato di risorse per la sussistenza. Pertanto, un ordinamento democratico che voglia essere e rimanere tale, deve: 1) annoverare norme di rango costituzionale attributive di questi diritti (non solo liberali ma anche) sociali; 2) sottrarre tali diritti al potere di revisione costituzionale; 3 ) introdurre norme legislative a garanzia dei medesimi.

Dal punto di vista della condizione di salvaguardia o sopravvivenza della democrazia, questi specifici diritti fondamentali, sia liberali sia sociali, insieme ai diritti politici di partecipazione alla formazione delle decisioni collettive, dovrebbero essere considerati diritti «supercostituzionali», come suggeriva Piero Calamandrei già nel 1946, ${ }^{18}$ cioè per l'appunto sottratti al potere di revisione costituzionale. Ma non già perché siano riconoscibili come (presunti) «diritti naturali»; ${ }^{19}$ bensì perché valgono nel loro complesso come precondizioni (diritti liberali e sociali) e condizioni (diritti politici) necessarie della democrazia.

d) Ma affinché sia soddisfatta la condizione di sopravvivenza della democrazia, enunciata sinteticamente (e riduttivamente) nella sesta regola della tavola di Bobbio, non basta neppure inserire questo nucleo di diritti fondamentali, sia liberali sia sociali, oltre ai diritti politici, nel «terreno proibito» di fronte a cui si deve arrestare la decisione di qualunque maggioranza. Dev'essere incluso nel coto vedado anche l'altro pilastro essenziale del costituzionalismo, indicato nelle clausole generalissime fissate dall'art. 16 della Dichiarazione francese del 1789: dunque, non solo quei diritti la cui garanzia è indispensabile al gioco democratico, perché ne sono le precondizioni e le condizioni; ma anche una qualche forma di divisione ed equilibrio dei poteri, insomma un complesso di tecniche idonee a prevenire e arginare il dispotismo, incluso quello della maggioranza. Da questo punto di vista, la salvaguardia della democrazia dipende anzitutto dalla separazione tra i poteri - cioè, tra gli organi e le funzioni - dello stato. ${ }^{20} \mathrm{O}$ meglio, dipende dalla solidità di architetture costituzionali capaci di assicurare: 1) il principio di legalità, ossia la distinzione e subordinazione della funzione esecutiva e di quella giudiziaria rispetto alla funzione legislativa; 2) il principio di imparzialità, ossia la separazione e l'indipendenza dell'organo giudiziario dall'esecutivo e dal legislativo.

e) Ma non basta ancora. Perché la democrazia possa nascere, continuare ad esistere e non degenerare in una qualche forma di autocrazia mascherata, è anche necessario salvaguardare quella che, seguendo Bobbio, considero la struttura più profonda, direi la costituzione materiale

18 P. Calamandrei, L'avvenire dei diritti di libertà, introduzione alla seconda ediz. di F. Ruffini, Diritti di libertà, La Nuova Italia Editrice, Firenze 1946, p. LIV.

19 Così invece li qualificava lo stesso Calamandrei (ibid.).

20 Per il problema dell'articolazione dei poteri dello stato, faccio riferimento alla sistemazione concettuale proposta da R. Guastini, Separazione dei poteri o divisione del potere?, in «Teoria politica», XIV, n. 3, 1998, pp. 25-42. Ma ora l'intera materia è stata sottoposta ad un trattamento teorico innovativo da Luigi Ferrajoli in Principia iuris cit., $\S \S 12.5-7$, che richiederà una considerazione più attenta e meditata in altra occasione. 
del costituzionalismo, ossia la separazione tra quelli che Bobbio chiama i tre «poteri sociali»: il potere politico, basato sul controllo dei mezzi di coazione; il potere economico, basato sul controllo dei beni e delle risorse materiali; e il potere ideologico, basato sul controllo delle idee e delle conoscenze, cioè dei mezzi di informazione e persuasione. ${ }^{21}$ La precondizione di tutte le precondizioni della democrazia, la clausola preliminare di un patto di convivenza democratica consiste nel divieto di concentrazione fra questi tre poteri.

5. Concludo in sintesi e schematicamente in due punti, lasciando spazio ad ulteriori futuri sviluppi argomentativi.

a) Il concetto ferrajoliano di «sfera dell'indecidibile» costituisce un avanzamento sia rispetto a quello di «coto vedado» di Garzón Valdés, sia a quello di «territorio inviolabile» di Bobbio, anzitutto perché è esplicitamente articolato in due parti o, potremmo dire continuando con la metafora, in due emisferi: seguendo formule di Ferrajoli, 1) «l'indecidibile che», e 2) «l'indecidibile che non». ${ }^{22}$ Il primo coincide con il divieto di limitare o sopprimere i diritti di libertà; ma non vedo perché non affiancare ad essi, nel medesimo emisfero, i diritti politici. ${ }^{23}$ Il secondo consiste nell'obbligo per i pubblici poteri, dunque per il potere politico di maggioranza, di introdurre specifiche e idonee garanzie legislative per (tutti i diritti fondamentali, ma specificamente per) i diritti sociali. Insomma, nella prospettiva di Ferrajoli diviene chiaro ed esplicito che il coto vedado non è solo vedado, il territorio inviolabile non è solo inviolabile: non contiene solo divieti, cioè l'indicazione normativa di quello che non si può decidere, bensì anche obblighi positivi, ossia l'indicazione normativa di quello che non si può non decidere.

Beninteso, anche per Garzón Valdés il coto vedado presenta due aspetti: «la prohibición de politizar lo no negociable y la exigencia de asegurar politicamente la realización efectiva de los derechos que intenta proteger». ${ }^{24}$ Tuttavia, nello stesso testo da cui cito Garzón Valdés precisa che il coto vedado «garantiza el ejercicio de la autonomía personal, sin intervención del Estado [...] protege el ámbito de la "privacidad"». ${ }^{25}$ In sostanza, l'idea sembra rimanere legata all'ispirazione del costituzionalismo liberale. E' vero che poco oltre l'autore considera la «posibilidad de expansión» del coto vedado verso i diritti di seconda o terza generazione; ma afferma che la via da seguire consiste nel «derivar nuevas conclusiones que están

21 Cfr. TGP, pp. 104-5, 167-72.

22 Per i luoghi dell'opera di Ferrajoli in cui compare la nozione e la sua articolazione in due emisferi, v. qui sopra, n. 4.

23 Nel testo di Principia iuris noto qualche esitazione su questo punto: non sempre l'inclusione dei diritti politici nella (emi-) sfera dell' «indecidibile che», insieme ai diritti di libertà, è richiamata esplicitamente.

24 E. Garzón Valdés, Para ir terminando cit., p. 48.

25 Ibidem. 
lógicamente implicadas en los principios y valores» già contenuti nel coto vedado. ${ }^{26}$

Quanto a Bobbio, le sue argomentazioni a favore dei diritti sociali sono ben note. ${ }^{27}$ Ma resta il fatto che, nell'affrontare lo specifico problema dei vincoli alla decisione democratica - che valgono al tempo stesso come limiti e pre-condizioni della medesima - il richiamo esplicito di Bobbio è ai diritti di libertà. E' forse questa sorta di (maggiore, se non unilaterale) accentuazione su tali diritti che porta sia Bobbio, sia Garzón Valdés a privilegiare la dimensione negativa dei vincoli costituzionali al potere democratico, insomma il lato del divieto, dell'inviolabile. In Ferrajoli, l'articolazione della nozione di «sfera del non decidibile», corrispondente a quelle di coto vedado e di «territorio inviolabile», in due emisferi (per così dire) equilibrati consente in particolare di cogliere la specificità del problema dei diritti sociali, e al tempo stesso di affermare per questa classe di diritti l'egual funzione di vincolo al potere democratico.

b) La concezione di Garzón Valdés assegna al coto vedado un valore morale: l'autore lo definisce come «ámbito consitucionalmente especificado que incluye principios y valores vinculados con bienes espirituales y materiales primarios». ${ }^{28} \mathrm{La}$ determinazione del suo contenuto e la sua forza vincolante sono affidate alla «morale critica». Dico subito che condivido le convinzioni morali di Garzón Valdés. Non quelle metaetiche. Come impenitente non-cognitivista, per un verso non credo che esistano «valori oggettivi», né che la morale critica (o meglio, il ragionamento morale) possa condurre per ogni questione ad una e una sola «risposta giusta»; per altro verso, non credo che il diritto debba proporsi il compito di esprimere e «dar forza» ad una morale, tanto meno alla morale (ad un codice morale determinato).

La concezione di Ferrajoli attribuisce alla sfera dell'indecidibile un significato e un valore vincolante eminentemente e genuinamente giuridico. Ma ciò comporta che la determinazione del suo contenuto resti affidata alle sorti delle battaglie politiche e ai processi di positivizzazione delle istanze ideali (o degli interessi materiali) che in esse di volta in volta storicamente prevalgono. Invece, le cinque regioni dell'indecidibile - nel duplice senso ferrajoliano, negativo e positivo - che ho cercato di delineare in base ad una interpretazione estensiva della sesta regola di Bobbio possono contare, mi sembra, anche su un fondamento razionale: sono determinabili in modo cogente per inferenza o per presupposizione a partire da un concetto formale di democrazia.

In questa prospettiva teorica, l'assunzione di un concetto formale di democrazia

\footnotetext{
26 Ivi, p. 49.

27 V. ad es. TGP, pp. 458-66.

28 E. Garzón Valdés, Para ir terminando cit., p. 43.
} 
come quello di Bobbio - ovvero, la scelta iniziale di definire la democrazia come «forma di regime» identificata da un determinato insieme di regole di competenza e di procedura, che riguardano il chi e il come, ossia appunto la forma, non il che cosa, cioè il contenuto, della decisione collettiva - è il necessario punto di partenza di un ragionamento che conduce, se correttamente svolto, ad una concezione della democrazia non puramente formale, bensì anche (in un certo senso, che non è quello più consueto negli usi storici di questa formula) sostanziale, in quanto individua i vincoli (costituzionali) di contenuto che debbono essere rispettati dalle decisioni democratiche al fine di preservare la stessa democrazia. In altre parole: per questa via, da un concetto formale si giunge ad una concezione costituzionale della democrazia. In piena sintonia finale, mi pare, con la visione di Luigi Ferrajoli.

\section{Bibliografia}

Bobbio, N. (1976). Democrazia, in Bobbio, N., Matteucci, N. (eds.), Dizionario di Politica, Torino, Utet.

(1976). Quale socialismo?, Torino, Einaudi.

. (1981). La regola di maggioranza: limiti e aporie, in «Fenomenologia e società», IV, 13-14.

. (1981). La regola della maggioranza e i suoi limiti, in AA.VV., Soggetti e potere, Napoli, Bibliopolis.

. (1984). Il futuro della democrazia, Torino, Einaudi.

. (1999). Teoria generale della politica, a cura di M. Bovero, Torino,Einaudi.

Bovero, M. (2003). Il concetto di democrazia. Per una ridefinizione radicale, in «Il Ponte», LIX, 2.

. (2005). Ma la democrazia ha un futuro? Uno sguardo dall'Italia, in «Ragion pratica», 25 .

. (2008). Isto é democracia?, in F. Schüler, G. Axt, J. Machado da Silva (eds.), Fronteiras do pensamento. Retratos de um mundo complexo, São Leopoldo, Editora Unisinos. . (2009). La democrazia e le sue condizioni, Modena, Notizie editrice. .(2010). Democrazia al crepuscolo?, in M. Bovero, V. Pazé(eds.), La democrazia 
in nove lezioni, Laterza, Roma-Bari.

. (2015). Para uma teoria neobobbiana da democracia, São Paulo, FGV Direito SP.

Calamandrei, P. (1946). L'avvenire dei diritti di libertà, in F. Ruffini, Diritti di libertà, Firenze, La Nuova Italia Editrice.

Ferrajoli, L. (2001). Diritti fondamentali, a cura di E. Vitale, Roma-Bari, Laterza. . (2007). Principia iuris. Teoria del diritto e della democrazia, 3 voll., RomaBari, Laterza.

Garzón Valdés, E. (2000). Instituciones suicidas, México, Paidós. . (2003). Para ir terminando in M. Atienza, El derecho como argumentación, México, Fontamara .(2005). El futuro de la democracia. Problemas conceptuales y empíricos: algunas propuestas de Norberto Bobbio, in L. Córdova Vianello, P. Salazar Ugarte (coordinadores), Politica y derecho. (Re)pensar a Bobbio, México, Unam \& Siglo ventiuno editores.

Guastini, R. (1998). Separazione dei poteri o divisione del potere?, in «Teoria politica», XIV, 3.

SOBRE O AUTOR:

Michelangelo Bovero

Professor de Filosofia da Universidade de Turim. Autor, dentre outras obras, de: Società e Stato nella filosofia politica moderna (com Norberto Bobbio, 1979), La democrazia e le sue condizioni (2009) e Para uma teoria neobobbiana da democracia (2015).

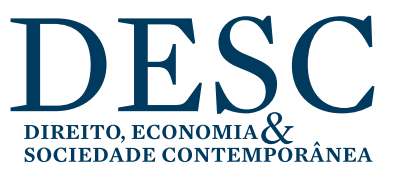

